\title{
Opioidergic Interactions between Striatal Projection Neurons
}

\author{
Craig P. Blomeley and Enrico Bracci \\ Faculty of Life Sciences, University of Manchester, Manchester M13 9PT, United Kingdom
}

Medium spiny striatal projection neurons (MSNs) release opioid neuropeptides, but the role of these neurotransmitters is still poorly understood. While presynaptic inhibition of corticostriatal axons by opioid receptors has been demonstrated using exogenous ligands, the action of synaptically released opioids in the striatum has not been investigated. We performed single and paired whole-cell recordings from rat MSNs while corticostriatal fibers were electrically activated. In single recording experiments, we also activated antidromically the axons of a population of MSNs. Corticostriatal fibers were stimulated once every $10 \mathrm{~s}$ and every other stimulation was preceded by 5 antidromic spikes (at $100 \mathrm{~Hz}$ ). This burst of antidromic spikes produced robust inhibition of evoked corticostriatal responses. This inhibition was not affected by the $\delta$-opioid receptor antagonist $S D M 25 \mathrm{~N}$, but was completely abolished by the $\mu$-opioid receptor antagonist CTOP. Inhibitory effects were maximal (on average $29.6 \pm 11.4 \%$ ) when the burst preceded the corticostriatal stimulation by $500 \mathrm{~ms}$ and became undetectable for intervals $>2 \mathrm{~s}$. Paired recordings from MSNs located $<100 \mu \mathrm{m}$ apart revealed that, in 30 of 56 (54\%) pairs, a burst of five action potentials in one of the MSNs caused significant inhibition (17.1 $\pm 5.7 \%)$ of evoked glutamatergic responses in the other MSN. In 5 of these pairs, reciprocal inhibition of corticostriatal inputs was present. These effects were maximal $500 \mathrm{~ms}$ after the burst and were completely blocked by CTOP. Thus, these results reveal a novel, strong opioid-mediated communication between MSNs and provide a new cellular substrate for competitive dynamics in the striatum.

\section{Introduction}

The striatum is the largest nucleus of the basal ganglia and is crucially involved in motor control, reinforcement learning, and action selection (Alexander and Crutcher, 1990; Graybiel et al., 1994; Redgrave and Gurney, 2006; Wickens et al., 2007b). Striatal neurons receive massive glutamatergic inputs from the cerebral cortex; this excitatory input is processed by a complex local network that converts it into a GABAergic inhibitory output directed to other basal ganglia nuclei. Striatal projection neurons are medium-sized spiny cells (MSNs) and make up $\sim 95 \%$ of the striatal neuronal population (Graveland and DiFiglia, 1985). Their axon collaterals form a dense local network in the striatum, where they form symmetric synapses with other MSNs; these synapses are primarily found near or on the shaft of dendritic spines that receive glutamatergic inputs, suggesting that they exert control over these excitatory signals (Wilson and Groves, 1980; Bolam and Izzo, 1988; Boyes and Bolam, 2007). Paired recording experiments have shown that GABAergic synapses between neighboring MSNs are functional (Tunstall et al., 2002; Koos et al., 2004; Shindou et al., 2008). In addition to GABA, striatonigral MSNs express substance $\mathrm{P}$ and dynorphin, while striatopallidal MSNs express enkephalin (Gerfen, 1992; Wang et

\footnotetext{
Received April 7, 2011; revised May 26, 2011; accepted July 14, 2011.

Author contributions: C.P.B. and E.B. designed research; C.P.B. performed research;C.P.B. and E.B. analyzed data;

C.P.B. and E.B. wrote the paper.

This study was funded by the Wellcome Trust (Grant n. 084706/Z/08/Z).

Correspondence should be addressed to Enrico Bracci, Faculty of Life Sciences, University of Manchester, AV Hill Building, Manchester M13 9PT, UK. E-mail: e.bracci@manchester.ac.uk.

DOI:10.1523/JNEUROSCI.1775-11.2011

Copyright $\odot 2011$ the authors $\quad 0270-6474 / 11 / 3113346-11 \$ 15.00 / 0$
}

al., 2006; Gertler et al., 2008). The function of these neuropeptides is less understood than that of GABA. Postsynaptic responses elicited in an MSN by action potentials in another MSN are fully abolished by $\mathrm{GABA}_{\mathrm{A}}$ receptor antagonists, suggesting that MSN-released neuropeptides do not mediate direct communication between MSNs (Tunstall et al., 2002; Tepper et al., 2008). Another potential role for these transmitters is modulation of cortical inputs. Using paired recordings and stimulation of corticostriatal fibers, we have recently shown that substance $\mathrm{P}$ released by MSNs strongly facilitates cortical glutamatergic inputs onto neighboring MSNs (Blomeley and Bracci, 2008; Blomeley et al., 2009). The ability of opioid neuropeptides released by MSNs to modulate cortical inputs has not been tested. Immunohistological experiments have shown that $\mu$ - and $\delta$-opioid receptors (MORs and DORs, respectively) are expressed throughout the striatum, although MORs are more densely expressed in the striatal patches (Miura et al., 2007). Presynaptic MORs and DORs are both present on glutamatergic terminals in the striatum (Wang and Pickel, 1998, 2001). Electrophysiological studies of opioids in the striatum were first performed by Jiang and North (1992), who showed that exogenous MOR and DOR agonists inhibit glutamatergic inputs to MSNs acting presynaptically. Further studies revealed that MOR activation inhibits corticostriatal responses to similar degrees in patch and matrix (Miura et al., 2007). Here, we tested the hypothesis that endogenous opioids released by MSNs inhibit the glutamatergic responses of neighboring MSNs, using an experimental approach similar to that used to investigate the effects of MSN-released substance $\mathrm{P}$ (Blomeley and Bracci, 2009). This protocol has the advantage of providing information on the extent and time 
course of the effects elicited by action potential-mediated release neurotransmitters on corticostriatal responses (Pakhotin and Bracci, 2007; Blomeley et al., 2009).

\section{Materials and Methods}

Slice preparation. Sprague-Dawley rats (14-29 d postnatal, both sexes) were killed by cervical dislocation in accordance with the UK Animals Act 1986; parasagittal brain slices (250-300 $\mu \mathrm{m}$ thick) were cut using a vibroslicer (Campden Instruments) and maintained at $25^{\circ} \mathrm{C}$ in oxygenated artificial CSF (ACSF) (in mm: $124 \mathrm{NaCl}, 2 \mathrm{KCl}, 1 \mathrm{MgSO}_{4}, 1.25$ $\mathrm{KH}_{2} \mathrm{PO}_{4}, 2 \mathrm{CaCl}_{2}, 26 \mathrm{NaHCO}_{3}$, and 10 glucose). For recordings, slices were submerged, superfused $(2-3 \mathrm{ml} / \mathrm{min})$ at $25^{\circ} \mathrm{C}$, and visualized with $10 \times$ and $40 \times$ water-immersion objectives (Olympus Optical) using standard infrared and differential interference contrast microscopy.

Whole-cell recordings. Paired and single whole-cell recordings were performed with $1.5 \mathrm{~mm}$ external diameter borosilicate pipettes, filled with intracellular solution (in mM: $120 \mathrm{~K}$-gluconate, $20 \mathrm{KCl}, 0.04 \mathrm{EGTA}$, 12 HEPES, $2 \mathrm{MgCl}_{2}, 4 \mathrm{Na}_{2} \mathrm{ATP}$, and $0.4 \mathrm{Na}_{2} \mathrm{GTP}$, adjusted to $\mathrm{pH} 7.3$ with $\mathrm{KOH})$. With this solution, pipette resistance was 3-4 M $\Omega$. Recordings in current-clamp mode were performed using bridge amplifiers (BA-01X, NPI). Data were acquired at $10 \mathrm{kHz}$ using Signal software and a micro1401 data acquisition unit (Cambridge Electronic Design). Input resistance was monitored under current-clamp conditions with small negative current injections (500 ms-1 s; $10-20 \mathrm{pA}$ ). In paired recording experiments, the distance between two MSNs was measured as the minimal distance between the somata of the cells as visible with the optics used. This is less than the distance between the centers of the cells, which could not be determined unequivocally.

Drugs. Drugs were all dissolved from freshly prepared stock solutions and bath-applied through the superfusion system. 2,3-Dihydroxy-6nitro-7-sulfonyl benzo[f]quinoxaline (NBQX) and AP-5 were obtained from Sigma-Aldrich; atropine sulfate, naloxone hydrochloride, $S-(-)$ sulpiride, SDM25N hydrochloride, CTOP, SCH 23390 hydrochloride, CGP 52432, SR 95531 hydrobromide (Gabazine), L-732,138 and tubocurarine chloride from Tocris Bioscience.

Evoked synaptic responses. Glutamatergic responses were recorded in current-clamp mode as EPSPs. These responses were evoked with a monopolar stimulating electrode, consisting of a patch micropipette filled with ACSF, placed either in the corpus callosum (CC), between the cortex and the striatum, or in the striatum just below $(<50 \mu \mathrm{m})$ the white matter, to activate preferentially corticostriatal fibers. This is referred to as CC stimulation. While this procedure is expected to preferentially activate corticostriatal axons, it is likely that some thalamostriatal fibers were also excited.

The resistance of the micropipettes used for stimulation was $0.3-1$ $\mathrm{M} \Omega$. Stimulation amplitude was $30-300 \mu \mathrm{A}$, and its duration was $100-$ $1000 \mu$ s. All the experiments were performed in the continuous presence of antagonists of the following receptors: $\mathrm{GABA}_{\mathrm{A}}$ (Gabazine, $10 \mu \mathrm{M}$ ); $\mathrm{GABA}_{\mathrm{B}}(\mathrm{CGP} 52432,2 \mu \mathrm{M})$; NK1 (L-732, 138, $\left.10 \mu \mathrm{M}\right)$; dopamine $\mathrm{D}_{1}$ (SCH 23390 hydrochloride, $10 \mu \mathrm{M}$ ); dopamine $\mathrm{D}_{2}(\mathrm{~S}$-(-)-sulpiride, 3 $\mu \mathrm{M}$ ), muscarinic (atropine sulfate, $25 \mu \mathrm{M}$ ), and nicotinic (tubocurarine chloride, $10 \mu \mathrm{M}$ ). Under these conditions, evoked responses, recorded under either current-clamp or voltage-clamp conditions, were completely abolished by coapplication of the ionotropic glutamate receptor antagonists NBQX $(10 \mu \mathrm{M})$ and AP-5 $(10 \mu \mathrm{M})(n=10)$.

In all experiments, a single electrical stimulus was applied every $10 \mathrm{~s}$. This interval was chosen because, after the first 10 stimuli, the evoked EPSP amplitude did not display a statistically significant trend of variation as a function of time (Blomeley et al., 2009). Since the first 3-10 responses were often significantly larger than the following ones, the first 10 responses were always discarded from analysis.

Single whole-cell recordings. In single whole-cell recording experiments, we also used a second stimulating electrode (similar to the one used to stimulate corticostriatal fibers) placed in the globus pallidus (GP) to antidromically activate MSN axons (Guzmán et al., 2003; Blomeley et al., 2009). Typical positioning of the stimulating and recording electrodes for these experiments is illustrated in Figure $1 \mathrm{~A}$. In these experiments, a large number (90-150 for each pharmacological condition) of
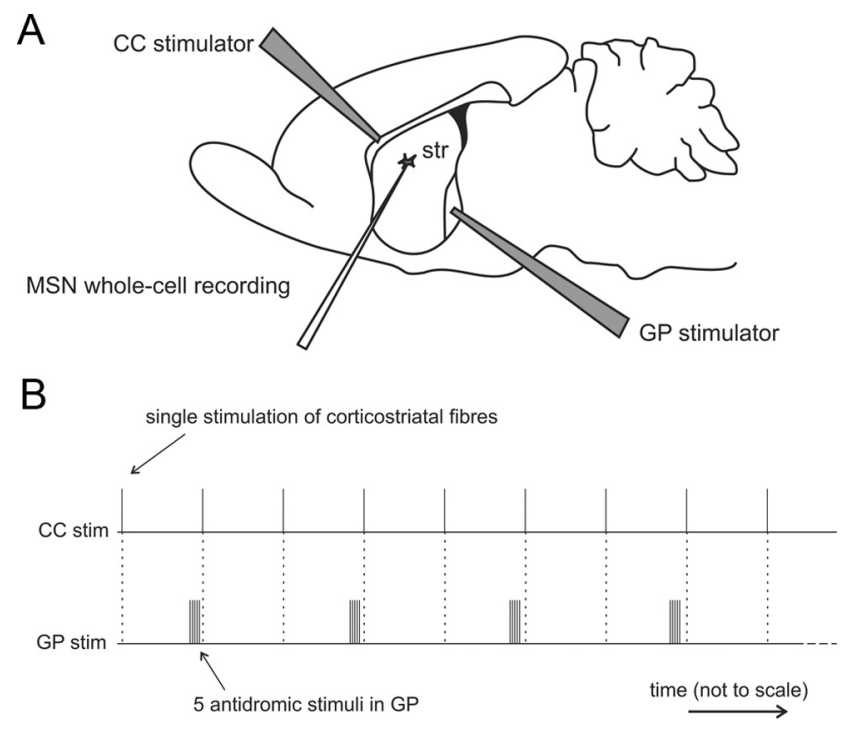

Figure 1. Experimental configuration used to investigate the effects of antidromic MSN activation on corticostriatal responses. $A$, Typical positioning of the patch and stimulating electrodes used to elicit orthodromic activation of corticostriatal fibers and antidromic activation of MSN axons in sagittal slices. Corticostriatal fibers were stimulated with an electrode placed in the $C C$, between the cortex and the striatum (str). MSN axons were stimulated with an electrode placed in the GP. B, Graphical illustration of the protocol used to investigate the effects of antidromic stimulation of a population of MSNs on the glutamatergic responses of the recorded MSN. For clarity, time is not to scale, as the intervals between single stimuli to corticostriatal fibers (10 s) have been compressed. Single C C stimuli (delivered every $10 \mathrm{~s}$ ) were sequentially preceded by: (1) no GP stimuli; (2) five GP stimuli; this two-step cycle was continuously repeated in each pharmacological condition.

single CC stimuli were continuously delivered every 10 s. Every other CC stimulation was preceded by a burst of GP five stimuli delivered at 100 $\mathrm{Hz}$. The interval between the first GP stimulus and the CC stimulus was varied between 0.5 and $3 \mathrm{~s}$. After each experiment, CC-evoked responses preceded or not preceded by GP stimuli were grouped for statistical analysis. This protocol in which the two conditions alternated every $10 \mathrm{~s}$ was used to prevent any subtle time-dependent change in EPSP amplitude from affecting the results. However, we continuously monitored the quality of the MSN recordings, and only the experiments in which the membrane potential and the input resistance did not change by $>5 \%$ during the whole experiment were accepted for analysis. A large number of observations were required because CC-evoked responses are intrinsically rather variable (typical coefficient of variation of evoked EPSP amplitude being $>20 \%$ ).

In some experiments we tested two different intervals (again defined as the temporal distance between the first GP stimulus and the CC stimulus). In these experiments, single CC stimuli were still delivered continuously every $10 \mathrm{~s}$ and were preceded (sequentially) by: no GP stimulation, GP stimulation at interval 1, GP stimulation at interval 2. Evoked responses for each condition were then grouped and compared statistically.

Spike-evoked response sequences. In paired recordings the two simultaneously recorded MSNs were arbitrarily named MSN1 and MSN2. Spikes were elicited in MSNs under current-clamp conditions by using short ( $3 \mathrm{~ms})$ but relatively large $(1.0-1.3 \mathrm{nA})$ current injections, separated by $10 \mathrm{~ms}$ intervals. This procedure allowed us to trigger bursts of spikes repetitively with high temporal precision (Blomeley et al., 2009). In these experiments, a single glutamatergic response was evoked every $10 \mathrm{~s}$ by stimulating the corticostriatal fibers. Each CC stimulus was sequentially preceded by (1) no spikes in MSN1 or MSN2 (control); (2) a burst of spikes in MSN1; (3) a burst of spikes in MSN2. This three-step sequence is illustrated below in Figure 6 and was repeated, without interruptions, 80-200 times for each pharmacological condition in each experiment. After an experiment, data for each condition $(1,2$, or 3$)$ were grouped for each pharmacological condition for statistical analysis. As 
for the single recording experiments, the continuous repetition of the three protocols ensured that any subtle time-dependent change would affect each condition equally. However, we continuously monitored the quality of the MSN recordings, and only the experiments in which the membrane potential and the input resistance of both cells did not change by $>5 \%$ during the whole experiment were accepted for analysis. The interval between the spikes in one MSN and the glutamatergic response of the other cell was measured from the start of the current injection that elicited the first spike of a burst to the time of the CC stimulation.

Statistical analysis. Values are expressed as mean $\pm \mathrm{SD}$, and statistical comparisons between two groups were made using MannWhitney test for unpaired data. We chose this nonparametric test as no assumption is needed about normality of data distribution. Statistical comparison of three groups of data was performed with Kruskal-Wallis test. Two populations of data points were considered to be significantly different if $p<0.05$. Statistical tests and cumulative frequency plots were implemented with OriginPro8 (OriginLab) and MATLAB software (The MathWorks).

\section{Results}

In total, 195 MSNs were used for this study. MSNs were identified by their distinctive electrophysiological properties (Kita et al., 1984; Nisenbaum et al., 1994) as in previous studies (Blomeley and Bracci, 2008; Blomeley et al., 2009). These properties included resting membrane potentials more negative than $-75 \mathrm{mV}$, strong inward rectification and delayed firing in response to depolarizing steps. The average resting membrane potential was $78 \pm 5 \mathrm{mV}$ and the average input resistance was $284 \pm 54 \mathrm{M} \Omega$.

To pharmacologically isolate evoked glutamatergic responses all the experiments were performed in the presence of $\mathrm{GABA}_{\mathrm{A}}$ receptor antagonists; furthermore, to limit the activation of other types of presynaptic receptors, the experiments were also performed in the presence of $G_{A B A}, N K 1$, dopamine $\left(D_{1}\right.$ and $\left.D_{2}\right)$, nicotinic, and muscarinic receptor antagonists (see Materials and Methods for details). Direct GABAergic connections between MSNs were pharmacologically blocked, and therefore could not be observed in these experiments.

\section{Antidromic action potentials in MSNs depress corticostriatal responses}

In parasagittal brain slices, stimulations delivered in the GP trigger antidromic spikes in a large population of MSNs, as the axons of both striatonigral and striatopallidal neurons pass through, or terminate in, this region (Guzmán et al., 2003; Blomeley et al., 2009). Such antidromic spikes will invade axon collaterals in the striatum and trigger release of neurotransmitters. Therefore, we used GP stimulation to elicit synchronized release of opioids from MSN axon collaterals while recording from a single MSN, while CC stimulation was used to activate corticostriatal afferents (see Materials and Methods and Fig. 1 for details of the stimulation protocol). All recorded MSNs were $>500 \mu \mathrm{m}$ from either stimulating electrode. The intensity of the GP stimulation was adjusted to $\sim 50 \%$ of the minimum intensity required to elicit antidromic spikes in the recorded MSN; this was chosen as a trade-off between the need to activate a large number of MSNs, and that to avoid antidromic spikes in the recorded MSN (which would have complicated the interpretation of the results). A relatively low intensity of stimulation was also important to limit the extent of the glutamatergic responses which were evoked in MSNs by GP stimulation, presumably as a result of antidromic activation of corticostriatal fibers directed toward the brainstem (Kincaid and Wilson, 1996), and orthodromic and/or antidromic activation of thalamostriatal fibers (Wilson et al., 1983). These GP-evoked EPSPs were not of interest in this study, but they could not be blocked as the integrity of glutamatergic signaling was essential in these experiments. The presence of GP-evoked EPSPs limited our ability to investigate the effects of GP stimuli on corticostriatal response evoked at intervals $<500 \mathrm{~ms}$ from the first GP stimulus (Blomeley et al., 2009). However, such intervals were investigated with paired recordings (as described below).

At 500 ms intervals, preceding GP stimulations significantly $(p<0.05)$ depressed the amplitude of CC-evoked responses in all MSNs tested (27 of 27). On average, the amplitude of CC-evoked EPSPs preceded by GP stimuli was $70.4 \pm 11.4 \%$ of control (i.e., responses not preceded by GP stimuli). Examples of this phenomenon are shown in Figures 2 and 3.

Exogenous agonists for MORs and DORs [but not for $\kappa$-opioid receptor (KOR)] decrease the amplitude of evoked glu- 
A

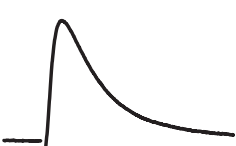

cc stim

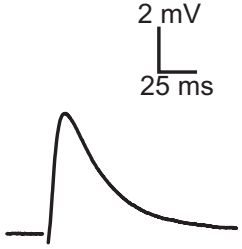

cc stim

B

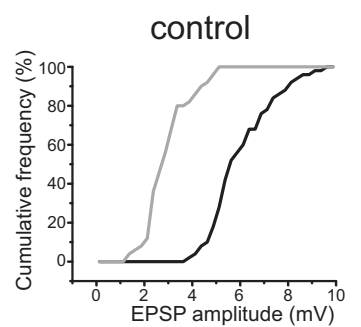

control

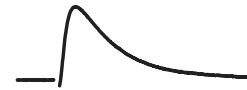

cc stim preceded by GP stims

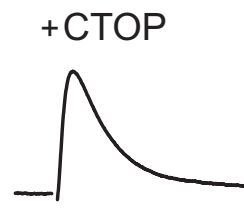

cc stim preceded by GP stims

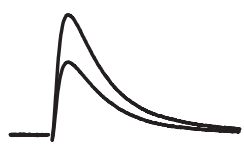

superimposed

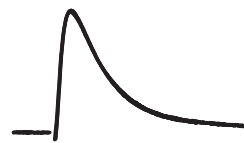

superimposed

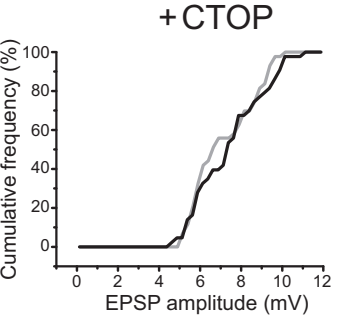

C

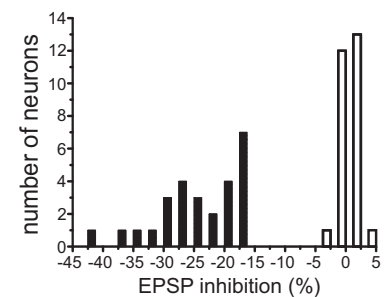

Figure 3. Antidromic MSN activation causes a CTOP-sensitive leftward shift in the cumulative distribution of evoked corticostriatal EPSP. $\boldsymbol{A}$, In this representative experiment, five preceding GP stimuli strongly decreased $(p<0.001)$ the amplitude of CC-evoked responses in a MSN. Each trace is the average of 100 consecutive trials consisting of either a single CC stimulation (left) or a single CC stimulation preceded by five GP stimuli (middle; the preceding GP stimuli are not shown). Average responses evoked by CC stimuli in the two cases are superimposed on the right. Subsequent bath application of CTOP fully abolished the effects of GP stimulation on corticostriatal responses (traces are averages of 100 consecutive trials for each protocol performed in the presence of (TOP). B , Cumulative distribution of CC-evoked EPSP amplitude for the MSN in A, in the absence (black line) or presence (gray line) of preceding GP stimulation, before (left) and after (right) application of CTOP. In the absence of CTOP, preceding GP stimulation causes a substantial leftward shift of the cumulative distribution of EPSP amplitude. These effects are abolished by CTOP. $\boldsymbol{C}$, Histogram illustrating the overall distribution of the inhibitory effects of five preceding GP stimuli on CC-evoked responses ( $500 \mathrm{~ms}$ interval) before (white columns) and after (black columns) application of CTOP. Data are from 27 experiments in which significant effects were observed before CTOP application. In each case these effects were abolished by CTOP.

tamatergic responses in MSNs (Jiang and North, 1992). Therefore, we investigated whether the depression of corticostriatal responses induced by preceding GP stimuli was affected by DOR and MOR selective antagonists.

In a series of experiments in which significant effects were observed in control solution, we applied sequentially the DOR antagonist SDM25N (500 nM) and the MOR antagonist CTOP (1 $\mu \mathrm{M})$ and repeated the stimulation procedure in the presence of these drugs. At least 80 CC stimuli were delivered in each pharmacological condition.

Typical results of these experiments are illustrated in Figure 2 (in which average responses are presented for each condition). In the presence of SDM25N, the GP stimulation-induced depression of CC-evoked responses persisted. In fact, in 6 of 6 cases, this depression was not significantly different in control solution $(22.7 \pm 3.4 \%)$ and in the presence of SDM $25 \mathrm{~N}(21.8 \pm 2.2 \%)$. Conversely, subsequent application of CTOP (still in the presence of SDM25N) fully blocked the GP stimulation-induced depression of CC-evoked responses in all cases.

In another 27 experiments, in which significant $(p<0.05)$ depression was observed in control solution, we applied CTOP in the absence of SDM25N. In all cases, CTOP fully blocked the inhibitory effects of GP stimulation on CC-evoked responses; a representative example of these experiments is illustrated in Figure 3. Figure $3 A$ shows the average responses before and after CTOP application, while Figure $3 B$ shows the cumulative distribution of CCevoked EPSP amplitudes for the experiments of Figure $3 A$ before (left) and after (right) application of CTOP. In control solution, preceding GP stimuli caused a large leftward shift in the amplitude distribution, which corresponded to a significant $(p<0.001)$ reduction. Conversely, in the presence of the MOR antagonist, the amplitude distributions for the response preceded or not preceded by GP stimuli were similar, and the average responses were not significantly different. The overall distribution of the effects of preceding GP stimuli on the amplitude of CCevoked EPSPs across the MSNs tested is illustrated in Figure 3C. In control solution, inhibitory effects spanned between $15 \%$ and $45 \%$, while in the presence of CTOP the effects are nonsignificant and distributed around zero. We concluded from these pharmacological experiments that the depression of glutamatergic responses caused by preceding GP stimulation was entirely due to the activation of MORs (presumably by enkephalin released from MSN terminals) and that DORs did not play a significant role in this phenomenon.

Application of MOR agonists depresses glutamatergic responses of MSNs (Jiang and North, 1992; Miura et al., 2007). We therefore compared the effects of endogenously released opioids with those induced by application of DAMGO $(1 \mu \mathrm{M})$, a selective MOR agonist. In 6 experiments, the effects of preceding GP stimulation on CC-evoked EPSP were observed in MSNs in control solution and, subsequently, in the presence of DAMGO. In all cases, preceding GP stimuli caused significant $(p<0.005)$ depression of CC-evoked responses $(16.1 \pm 4.5 \%)$, similar to the experiments described above. Application of DAMGO caused a significant depression of CC-evoked EPSPs (not preceded by GP stimuli) in all cases (on average, by $19.4 \pm 3.9 \%$ ); furthermore, in the presence of DAMGO the depressing effects of preceding GP stimuli on CCevoked EPSPs were completely absent in all cases. A representative example of these experiments is shown in Figure $4 A$. In 2 of 6 MSNs, the effects of DAMGO on CC-evoked EPSPs were significantly $(p<0.001)$ larger than those caused (in the absence of DAMGO) by preceding GP stimuli. In the remaining 4 of 6 cases, the effects of DAMGO on were not significantly different from those of preceding GP stimuli.

Endocannabinoids play an important role in the control of glutamatergic inputs to MSNs (Lovinger, 2010). Therefore, we tested whether the present phenomenon depended on the activation of CB1 receptors. In 5 experiments in which preceding GP stimuli caused significant depression $(20.5 \pm 2.8 \%)$ of CCevoked EPSPs in MSNs in control solution, subsequent application of the selective CB1 receptor antagonist AM251 (3 $\mu \mathrm{M})$ failed 
to affect significantly either the amplitude of the CC-evoked responses or the depressing effects caused by preceding GP stimuli. A representative example of these experiments is shown in Figure 4B. We concluded that the depression of CC-evoked EPSP caused by preceding antidromic activation of MSNs did not depend on the activation of CB1 receptors.

We then investigated how these inhibitory effects mediated by MORs depended on the temporal distance between GP stimulation and activation of corticostriatal fibers. To do this, it was essential to compare different GP-CC stimulation intervals in the same experiment. Given the nature of these experiments, which require a large number of trials for each condition, we tested only two intervals in the same experiment (see Materials and Methods for details of the procedure). As a reference interval, $500 \mathrm{~ms}$ was used and compared with intervals of 1,2 or $3 \mathrm{~s}$. An example of these experiments is shown in Figure 5, $A$ and $B$. In this case intervals of $500 \mathrm{~ms}$ and $2 \mathrm{~s}$ were tested. In control solution, a significant $(p<0.001)$ reduction in CC-evoked EPSPs was observed when GP stimuli preceded the CC stimuli by $500 \mathrm{~ms}$, but not by $2 \mathrm{~s}$. These effects are quantified by the cumulative distribution of CC-evoked EPSP amplitudes in Figure $5 B$, where a large leftward shift is present at $500 \mathrm{~ms}$ but not at $2 \mathrm{~s}$ intervals. Subsequent application of CTOP fully blocked the inhibitory effects of GP stimulation at $500 \mathrm{~ms}$ intervals (Fig. 5A, B). In a series of similar experiments, in which significant $(p<0.001)$ depression was present at 500 $\mathrm{ms}$ intervals, we found that significant $(p<0.05)$ depression was also found at $1 \mathrm{~s}$ intervals in 4 of 13 cases, but not at $2 \mathrm{~s}$ $(n=8)$ or $3 \mathrm{~s}(n=6)$. In all cases, these effects were blocked by subsequent application of CTOP. The average depression of CC-evoked responses as a function of the interval between GP and CC stimulation for 500, 1000, 2000, and $3000 \mathrm{~ms}$ in all MSNs tested is presented in Figure 5D. This plot shows that after a burst of GP stimuli there is an approximately exponential decay of the inhibitory effects, which are maximal at the shortest interval tested $(500 \mathrm{~ms})$, are reduced by approximately one third after $1 \mathrm{~s}$ and vanish at $2 \mathrm{~s}$.

\section{Action potentials in a single MSN reduce glutamatergic responses in neighboring MSNs}

The experiments performed with antidromic stimulation of MSN axons in the GP did not allow us to control the number of MSNs activated antidromically and causing inhibition of EPSPs. Can action potentials in single MSN exert inhibitory effects on corticostriatal inputs? To address this issue, we performed paired recordings from MSNs located $<100 \mu \mathrm{m}$ apart in sagittal brain

B
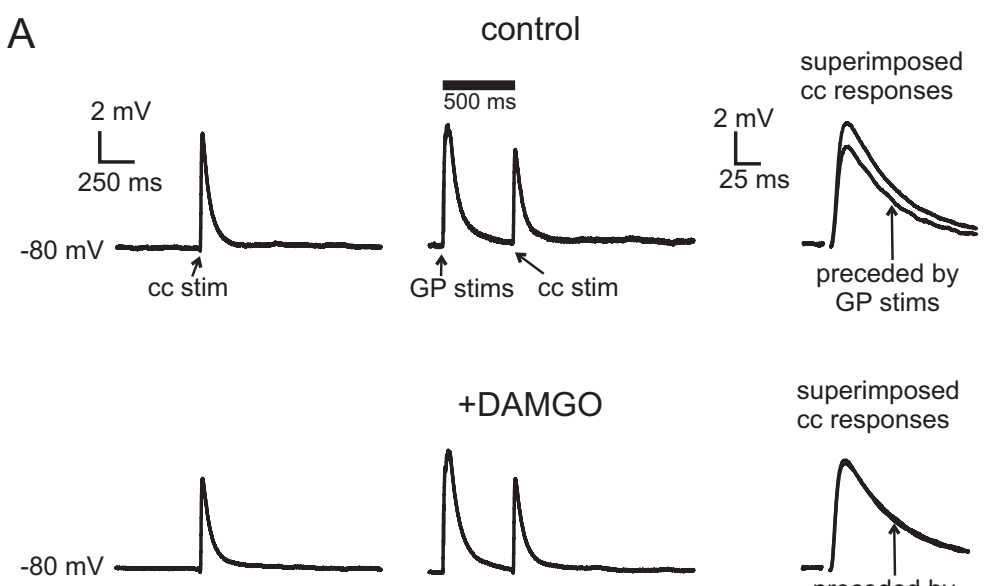

superimposed cc responses

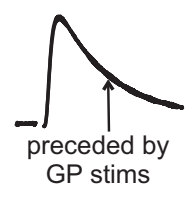

4. Depression of corticostriatal responses by antidromic MSN activation is mimicked and occluded by a MOR agonist and not affected by a CB1 receptor antagonist. $\boldsymbol{A}$, In this representative experiment, five preceding GP stimuli strongly decreased ( $p<$ $0.001)$ the amplitude of CC-evoked responses in a MSN. Each trace is the average of 100 consecutive trials consisting of either a (left) or a single CC stimulation preceded by five GP stimuli. Average responses evoked by CC stimuli in the two cases are superimposed on the right. Subsequent bath application of DAMGO $(1 \mu \mathrm{M})$ caused a significant $(p<0.001)$ reduction in the amplitude of the CC-evoked EPSPs and abolished the ability of preceding GP stimuli to affect CC-evoked EPSPs (traces are stimuli strongly decreased $(p<0.001)$ the amplitude of CC-evoked responses in a MSN. Each trace is the average of 100 consecutive trials consisting of either a single CC stimulation (left) or a single CC stimulation preceded by five GP stimuli. Average responses evoked by CC stimuli in the two cases are superimposed on the right. Subsequent bath application of AM251 (3 $\mu \mathrm{M}) \mathrm{did}$ not affect the CC-evoked EPSPs or the ability of preceding GP stimuli to depress them (traces are averages of 100 consecutive trials for each protocol performed in the presence of AM251).

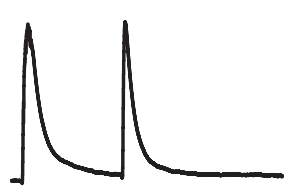

slices. All recorded cells were $>500 \mu \mathrm{m}$ from the stimulating electrode placed in the CC. The typical positioning of the recording and stimulating electrodes is shown in Figure 6A. In these experiments, a single CC stimulation was continuously delivered every $10 \mathrm{~s}$; these CC stimuli were sequentially preceded by: (1) no spikes in MSN1 or MSN2; (2) a burst of five spikes in MSN1; (3) a burst of 5 spikes in MSN2. This protocol is illustrated in Figure $6 \mathrm{~B}$ and described in detail in the Materials and Methods. Each spike was elicited in a temporally precise fashion using $3 \mathrm{~ms}$ current steps (Blomeley et al., 2009). The interval was defined as the temporal distance between the first spike in a MSN and the CC stimulus. 
A
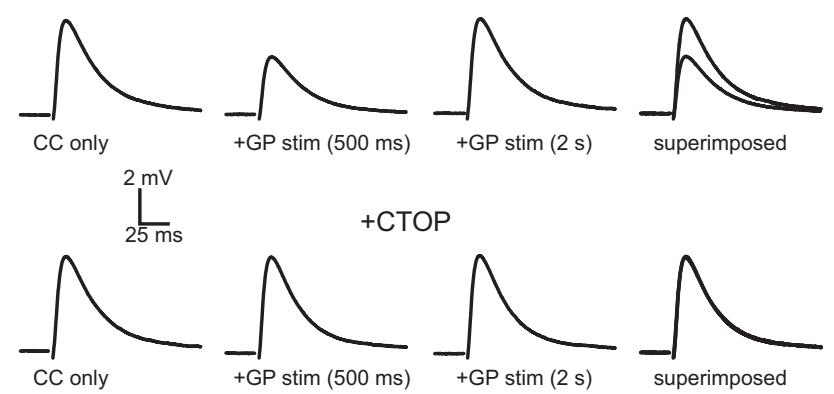

B

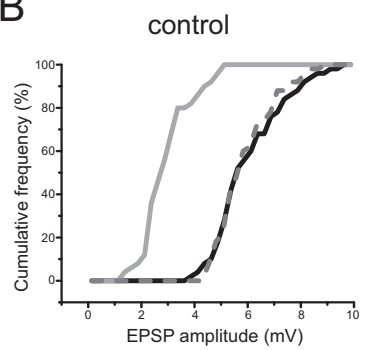

C

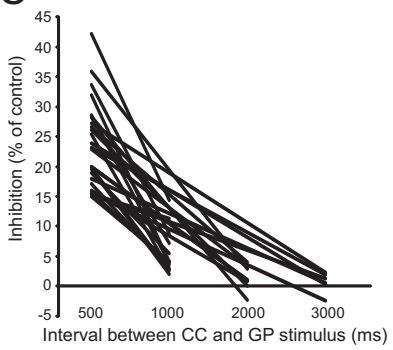

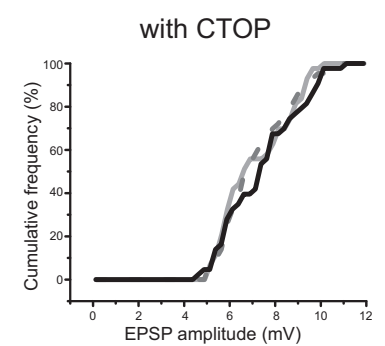

D

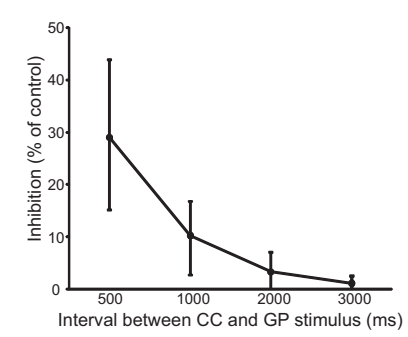

Figure 5. Depression of corticostriatal responses induced by GP stimulation is maximal after $500 \mathrm{~ms}$. $A$, In this representative experiment, a single CC stimulus was delivered every $10 \mathrm{~s}$. These CC stimuli were sequentially preceded by: (1) no GP stimulation; (2) five GP stimuli preceding $\mathrm{CC}$ stimulation by $500 \mathrm{~ms}$; and (3) five GP stimuli preceding $\mathrm{CC}$ stimulation by $2 \mathrm{~s}$. Traces are averages of 90 consecutive trials for each protocol. GP stimuli significantly $(p<$ 0.001 ) inhibited C(-evoked responses at $500 \mathrm{~ms}$, but not at 2 s intervals. $\boldsymbol{B}$, Cumulative distribution of CC-evoked EPSP amplitude for the MSN in $A$, in the absence (black line) or presence of preceding GP stimuli (solid gray line: $500 \mathrm{~ms}$ interval; dashed gray line: 2 s interval), before and after application of CTOP. In the absence of CTOP, preceding GP stimuli caused a substantial leftward shift of the cumulative distribution of EPSP amplitude at $500 \mathrm{~ms}$, but not $2 \mathrm{~s}$, interval. These effects were abolished by CTOP. C, In 27 different experiments, 500 ms intervals were compared with a different interval $(1,2$, or 3 s). For each experiment, data points representing average depression observed for the two intervals tested are connected by a line. In all cases, larger effects were observed at $500 \mathrm{~ms}$ then at the other interval tested. $\boldsymbol{D}$, Average effects of GP stimulation on CC-evoked responses as a function of the interval preceding CC stimulation. Data from all experiments in which significant effects were found for at least one interval are included. Effects are maximal at $500 \mathrm{~ms}$, and decay in an approximately exponential fashion.

We performed 56 paired recording experiments; the interval between the spikes triggered in one of the cells and the stimulation of corticostriatal fibers was $500 \mathrm{~ms}$ for both MSNs. In 30 of 56 pairs $(54 \%)$, spikes in one of the two MSNs caused a significant $(p<0.05$ or $p<0.001)$ decrease in the responses of the other MSNs; in the remaining 26 pairs, the presence of preceding spikes in either MSN did not significantly affect the evoked responses of the other MSN. MSN spikes did not cause significant facilitation of the evoked responses in any of the experiments. In 5 of 56 pairs, the inhibitory effects were bidirectional (i.e., each MSN depressed the evoked responses of the other MSN). A representative example of these bidirectional interactions is shown in Figure 7; average recordings for the three protocols described above are

A
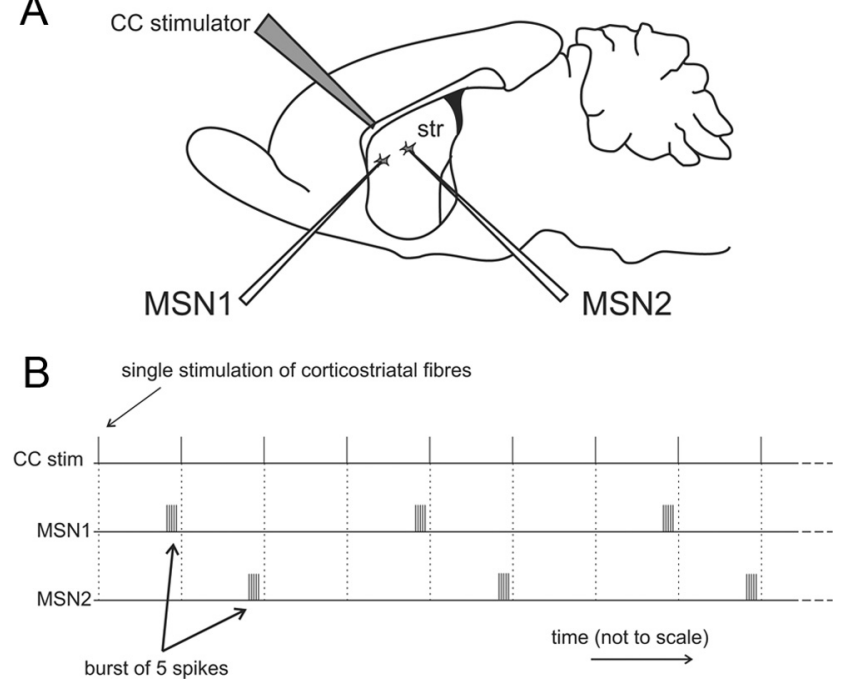

Figure 6. Experimental configuration for paired recording experiments. $\boldsymbol{A}$, Typical positioning of recording and stimulating electrodes used for paired recording experiments. A single stimulating electrode (gray) was used to activate corticostriatal fibers end evoke glutamatergic responses in MSNs. Simultaneously recorded MSNs were located $<100 \mu \mathrm{m}$ apart in the striatum (str). $\boldsymbol{B}$, Illustration of the protocol used to investigate the effects of spikes in each MSN on the glutamatergic responses of the other MSN. For clarity, time is not represented to scale, as the intervals between $C($ stimuli $(10 s)$ have been compressed. Single C $C$ stimuli (delivered every 10 s) were sequentially preceded by: (1) no spikes; (2) five spikes in MSN1; (3) five spikes in MSN2; this three-step cycle was continuously repeated in each pharmacological condition.

shown for each MSN, with average CC responses in the presence or absence of spikes in the other MSN are enlarged on the right. The inhibitory effects of MSN spikes were completely abolished by subsequent application of CTOP (Fig. 7B). Consistently, the cumulative distribution of evoked EPSP amplitudes for each MSN shows a large leftward shift when CC stimuli were preceded by spikes in the other MSN in control solution, but not in the presence of CTOP (Fig. 7C,D).

On average, when significant effects were observed, the reduction of CC-evoked EPSPs caused by MSN spikes was $17.1 \pm 5.7 \%$. In all cases in which significant effects of MSN spikes on CCevoked EPSPs were observed, these effects were abolished in the presence of CTOP.

In a different series of experiments, we used a similar protocol to investigate how MSN spike-induced effects depended on the temporal distance from the evoked EPSP. As in the case of GP-stimulation experiments, it was only possible to test two intervals in a given paired-recording experiment. Therefore, in each experiment, CC single stimuli were sequentially preceded by: (1) no spikes; (2) a burst of five spikes in MSN1 at interval 1; (3) a burst of five spikes in MSN2 at interval 1; (4) a burst of five spikes in MSN1 at interval 2; (5) a burst of five spikes in MSN2 at interval 2. As in the GP stimulation experiments, $500 \mathrm{~ms}$ was used as a reference interval and alternated with either $250 \mathrm{~ms}$ or $1 \mathrm{~s}$ intervals.

In 10 of $10 \mathrm{MSN}$ pairs in which significant $(p<0.001)$ effects were observed at $500 \mathrm{~ms}$ intervals (in one direction), no significant effects were observed at $250 \mathrm{~ms}$ intervals. A representative example of this finding is presented in Figure $8 A$. In another series of experiments, out of 18 pairs in which significant $(p<$ 0.05 ) depression was present at $500 \mathrm{~ms}$ intervals (in one direction), significant depression was also observed for $1000 \mathrm{~ms}$ intervals in 3 cases. In the remaining 15 cases, no significant effects on CC-evoked EPSPs were present at $1000 \mathrm{~ms}$ intervals. An example 
A
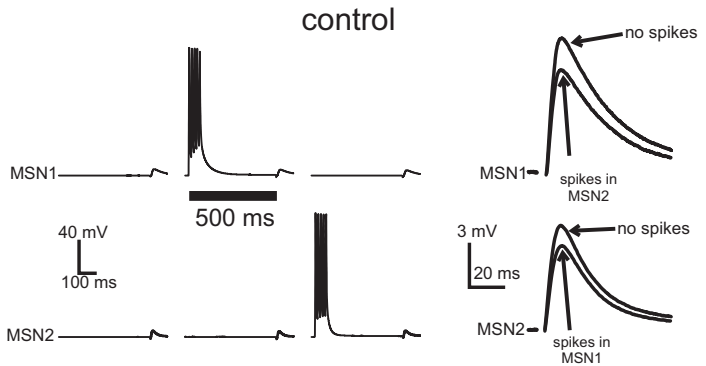

B
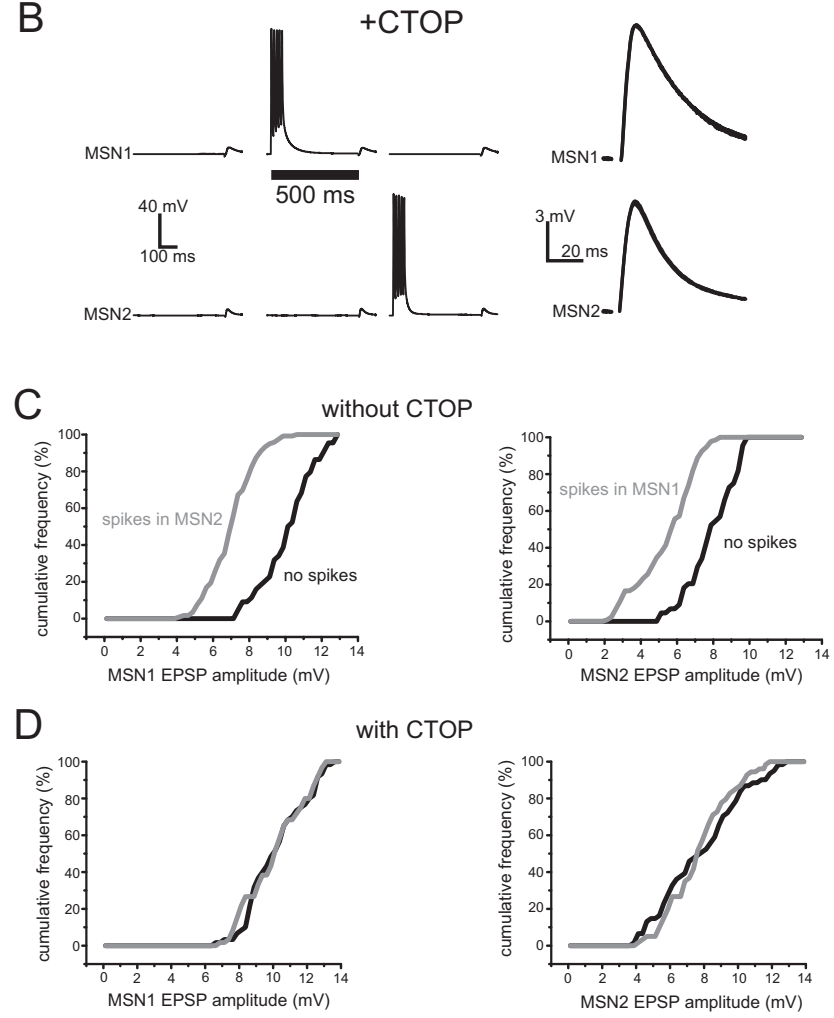

with CTOP

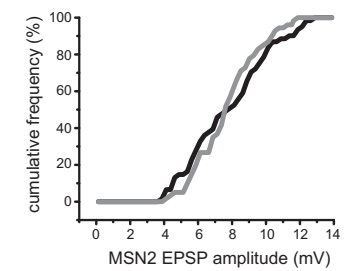

Figure 7. Action potentials in a single MSN depress glutamatergic responses of neighboring MSNs acting through MORs. A, Average responses of two simultaneously recorded MSNs to stimulation of corticostriatal fibers in the absence of preceding spikes (left); in the presence of a preceding burst of five spikes in MSN1 (middle); and in the presence of a preceding burst of five spikes in MSN2 (right). The first spike of a burst in MSN1 or MSN2 preceded the stimulation of corticostriatal fibers by $500 \mathrm{~ms}$. Each trace is the average of 180 consecutive trials for that protocol. Average evoked responses in MSN1 and MSN2 in the absence or presence of preceding spikes in the other cell are also magnified on the right. In this experiment, spikes in each MSN significantly $(p<0.001)$ depressed the evoked responses of the other MSN. $\boldsymbol{B}$, In the same experiment, in the presence of CTOP, depression of evoked responses was completely abolished in both MSNs. Each trace is the average of 150 consecutive trials for that protocol performed in the presence of CTOP. C, Cumulative distribution of evoked EPSP amplitude in the same MSNs of $\boldsymbol{A}$ and $\boldsymbol{B}$, in the absence (black line) or presence (gray line) of preceding spikes in the other MSN. In both cases, a large leftward shift of the distribution in the presence of spikes in the other cell is clearly visible. D, Cumulative distribution of EPSP amplitudes in the presence of CTOP. For both cells, the EPSP distributions with or without preceding spikes in the other cell are now similar, as CTOP fully blocked the inhibition of evoked responses.

of these results is illustrated in Figure $8 \mathrm{~B}$. Subsequent bathapplication of CTOP completely abolished these effects in all cases.

The overall picture emerging from these experiments is illustrated in Figure 9, which reports the results of individual experiments, as well as average results for each interval. This shows that the inhibitory effects of a burst of spikes in a single MSN on the cortical responses of another MSN are present in a limited and

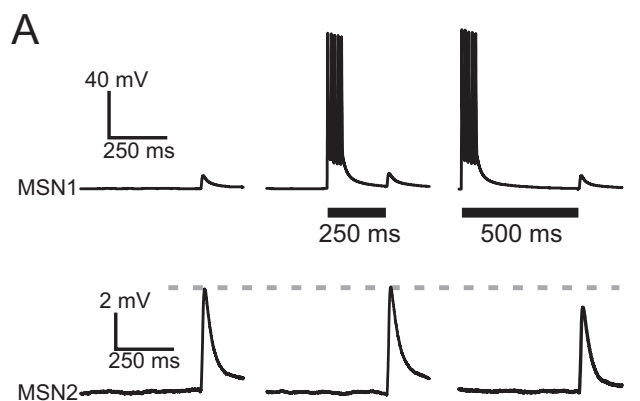

B

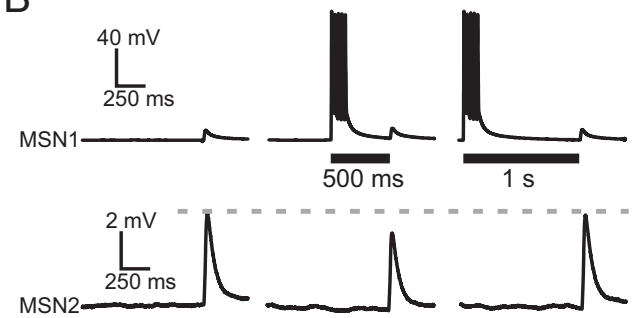

Figure 8. The inhibitory effects of MSN spikes on corticostriatal responses are maximal at 500 ms intervals. $\boldsymbol{A}$, In this experiment, a burst of 5 spikes in MSN1 significantly $(p<0.05)$ decreased EPSP amplitude in MSN2 when the interval between the first spike and the glutamatergic response was $500 \mathrm{~ms}$; however, no significant facilitation was present when this interval was $250 \mathrm{~ms}$. Traces are averages of 130 consecutive trials for each protocol. $\boldsymbol{B}$, In a different pair of MSNs, a burst of five spikes in MSN1 significantly $(p<0.05)$ depressed the evoked responses of MSN2 for an interval of $500 \mathrm{~ms}$ but not for an interval of $1000 \mathrm{~ms}$.

well defined time window, being undetectable after $250 \mathrm{~ms}$, peaking around $500 \mathrm{~ms}$ and being substantially reduced, or absent, after $1 \mathrm{~s}$.

\section{Corticostriatal responses differ in presynaptic and postsynaptic MSNs involved in opioid-mediated communication}

Recent studies using BAC transgenic mice expressing GFP in $D_{1}$ or $\mathrm{D}_{2}$ dopamine receptor-expressing MSNs have revealed that the corticostriatal EPSPs of striatopallidal MSNs have a significantly faster time course than those of striatonigral MSNs (Flores-Barrera et al., 2010). To gain insight into the identity of the MSNs involved in opioid-mediated communication, we tested whether the MSNs causing these effects through their action potentials (referred to as presynaptic MSNs) differed in these features from those receiving the inhibitory effects (referred to as postsynaptic MSNs). Overall, in pairs with significant interactions, the CC-evoked EPSPs observed in presynaptic and postsynaptic MSNs did not differ significantly in their amplitude; however, we noticed that in some of the experiments the timeto-peak and the half-width of evoked EPSP were larger in the postsynaptic MSNs; in the other experiments similar values were observed in presynaptic and postsynaptic MSNs. Two examples of these different situations are illustrated in Figure $10 \mathrm{~A}$, while paired (pre-post) comparisons for individual experiments are presented in Figure 10C. When responses from all presynaptic and postsynaptic neurons were grouped, significant differences were found for EPSP half-widths $(33.6 \pm 6.3 \mathrm{~ms}$ for presynaptic MSNs and $49.6 \pm 23.1 \mathrm{~ms}$ for postsynaptic MSNs; $p<0.001)$ and time-to-peak of the glutamatergic responses $(14.4 \pm 3.1 \mathrm{~ms}$ in presynaptic MSNs and $17.9 \pm 3.8 \mathrm{~ms}$ in postsynaptic MSNs; $p<$ $0.05)$, as shown in Figure $10 \mathrm{~B}$. These results suggested that the presynaptic MSNs belonged to the striatopallidal subpopulation while the postsynaptic MSNs were a mixture of striatopallidal 
A

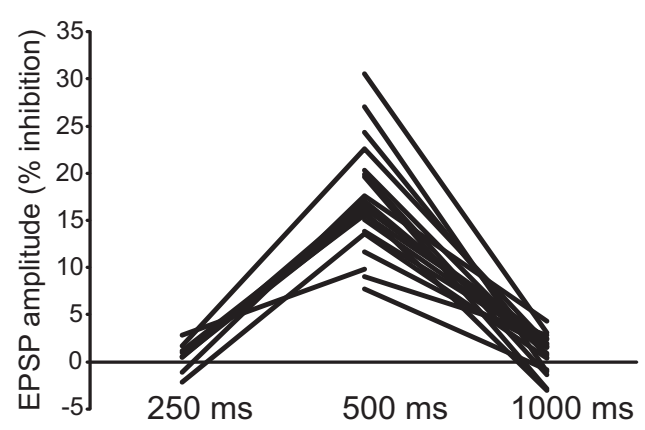

$\mathrm{B}$

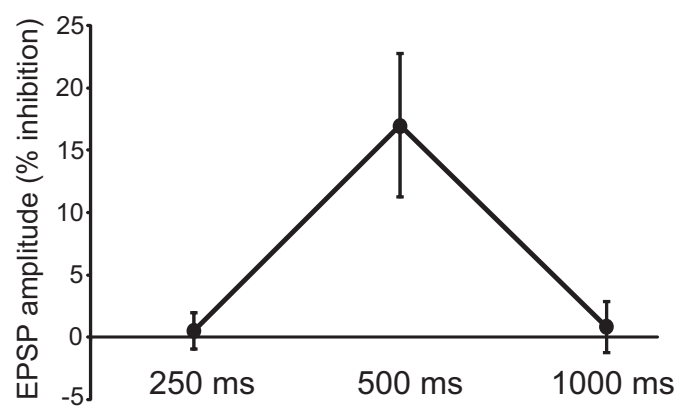

Figure 9. Depression of corticostriatal responses is maximal $500 \mathrm{~ms}$ after a burst of spikes. $\boldsymbol{A}$, Data were collected from paired recording experiment in which significant effects were present in at least one direction at $500 \mathrm{~ms}$ and another interval (either 250 or $1000 \mathrm{~ms}$ ) was tested. Data points representing average depression of $\mathrm{CC}$-evoked responses for two intervals in the same experiment are connected by a line. At $250 \mathrm{~ms}$ intervals, significant depression was never present. In 3 of 18 experiments there was significant $(p<0.05)$ depression at $1000 \mathrm{~ms}$, although this was always smaller than at $500 \mathrm{~ms}$. B, Average inhibitory effects at 250, 500 and 1000 ms intervals for the experiments of $\boldsymbol{A}$.

and striatonigral cells. Consistent with this notion, the distribution of EPSP half-width and time-to-peak had a wider extent for the postsynaptic than presynaptic MSNs (Fig. 10D).

\section{Discussion}

The present experiments have revealed a novel form of opioidmediated communication between striatal projection neurons. These cells were shown to inhibit the corticostriatal inputs to neighboring neurons acting through MORs, but not DORs. Using experimental protocols in which the effects of endogenous, spike-released opiates can be observed, we were able to characterize quantitatively both the extent and the time course of these interactions.

Previous experiments with exogenous agonists showed that both MOR and DOR (but not KOR) agonists suppress glutamatergic responses in MSNs, acting at a presynaptic level (Jiang and North, 1992). However, in the present experiments, the inhibition of glutamatergic inputs caused by MSN spikes was not affected by DOR antagonists. This suggests that DORs, while present and functional on glutamatergic terminals, are not easily reached by synaptically released peptides, possibly as a result of a more distal location from MSN terminals. On the other hand, the effects mediated by MORs in our experiments are likely to share the same presynaptic mechanism activated in the experiments by Jiang and North (1992) by exogenous MOR agonists. Consistent with this notion, the depressing effects of antidromic spikes in MSNs were mimicked and occluded by the MOR agonist DAMGO. Interestingly, in most cases the depression of evoked
EPSP caused by a saturating concentration of DAMGO was not larger than that caused by antidromic MSN activation in the absence of DAMGO. In these experiments the intensity of GP stimulation was far from maximal and was set to activate $\sim 50 \%$ of MSNs whose axons were in reach of the stimulator. It is likely that DAMGO desensitized MORs, as previously described in this system (Barral et al., 2003). Alternatively, the opioids released by MSNs may have saturated the MORs controlling the glutamatergic terminals activated by CC stimulation.

The differences between the effects of endogenous and exogenous ligands are instructive and suggest that caution should be exercised when interpreting the effects of exogenous agonists. The protocols developed by our group have allowed us to study presynaptic interactions between striatal neurons mediated by endogenous neurotransmitters (Pakhotin and Bracci, 2007; Blomeley et al., 2009). One methodological challenge in this kind of experiments is that the corticostriatal responses have an elevated degree of intrinsic variability. Typically, the amplitude of the EPSPs evoked at $0.1 \mathrm{~Hz}$ in healthy MSNs has a coefficient of variation (SD/average) of $10-20 \%$. This is of the same order of magnitude as the endogenous presynaptic modulation. It is therefore necessary to repeat the stimulation protocol many times to reveal statistically significant effects. One side-effect of these long experiments is that their duration makes the reconstruction of neurons injected with biocytin or neurobiotin very difficult (this procedure only succeeded when whole-cell recordings were $<45 \mathrm{~min}$ long). Another methodological issue concerns the identity of the fibers activated by CC stimulation. While it is reasonable to assume that these fibers were mostly corticostriatal axons, it is likely that some thalamostriatal axons have been stimulated as well (Smeal et al., 2007). Further studies will be required to establish whether MOR-mediated inhibition is differentially distributed in these two excitatory inputs to the striatum.

Paired recording experiments revealed important features of presynaptic inhibition between MSNs. In terms of connectivity, this form of communication appears to be rather prominent when compared with other MSN-MSN interactions. Communication in at least one direction was found here in 30 of 56 (54\%) of pairs. Koos et al. (2004) found that $12 \%$ of pairs of MSNs (tested unidirectionally) were connected by a GABAergic synapse (corresponding to $22.5 \%$ of pairs estimated to be connected in at least one direction); we previously found NK1 receptor-mediated facilitation of glutamate inputs (in at least one direction) in 23\% of MSN pairs (Blomeley et al., 2009).

The magnitude of the depressing effects of spikes in single MSNs was on average $58 \%$ of that observed with antidromic activation of a population of MSNs. However, the effects of a single MSN firing may have been overestimated due to a possible statistical bias. In fact, the effects caused by firing in a single MSN were averaged only for pairs in which significant effects were observed. Given the intrinsic variability of evoked EPSPs in MSNs, and the finite number of trials that can be performed in an experiment, it is possible that some pairs in which weak effects were present did not reach the significance threshold and were therefore not included in the averages.

MORs are activated with high affinity by enkephalin (Wee and Koob, 2010); it is therefore most likely that inhibition of corticostriatal inputs was caused by striatopallidal MSNs, which express this neuropeptide (in addition to GABA and $\mathrm{D}_{2}$ dopamine receptors; Gerfen, 1992). On the other hand, it is unlikely that striatonigral MSNs (that express dynorphin, GABA, substance $\mathrm{P}$ and $D_{1}$ dopamine receptors; Gerfen, 1992) caused these interactions 
by releasing dynorphin, given the marked preference of this opioid for KORs (Wee and Koob, 2010). Action potentials in striatopallidal MSNs are expected to invade local axon collaterals and release enkephalin, which is found in presynaptic terminals forming synaptic contacts on MSN dendrites (Yung et al., 1996). Our results suggest that enkephalin diffused in the extracellular space and bound presynaptic receptors on corticostriatal terminals; cortical synapses are mostly located on MSN dendritic spines, close to GABAergic synapses (Boyes and Bolam, 2007). Alternatively, activation of MORs may trigger the release of another neurotransmitter responsible for the depression of glutamate responses. For instance, the involvement of endocannabinoids in this phenomenon was a plausible hypothesis, given their ability to depress corticostriatal synapses with relatively fast time course acting on CB1 receptors (Lovinger, 2010). However, our experiments with a CB1 receptor antagonist clearly showed that MOR-dependent depression of glutamatergic responses did not require the activation of these receptors.

An interesting issue is raised by the observation that MSNs retained their ability to depress glutamatergic inputs when they were activated every $10 \mathrm{~s}$ for long periods. Thus, these cells must be able to replenish effectively the neuropeptides released during this relatively frequent activity. Further experiments will be required to determine whether this is accomplished trough active axonal transport or simply by a large reserve of releasable neuropeptide.

Which MSNs were the targets of MORmediated depression of cortical inputs? In single whole-cell recording experiments, MOR-dependent inhibitory effects were observed in 27 of 27 MSNs. This observation strongly suggests that corticostriatal inputs to both striatopallidal and striatonigral MSNs were inhibited by endogenous enkephalin: the probability that all the 27 recorded MSNs belonged to only one of the two subpopulations is extremely low: $(1 / 2)^{27}=0.000000745 \%$. As mentioned above, the long duration of these experiments prevented us from carrying out post hoc immunohistochemical procedures on biocytin-filled MSNs. However, the notion that postsynaptic MSNs belonged to a mixed population is further supported by the observation that the half-width and the time-to-peak of the evoked EPSPs had a narrower distribution, and a smaller average value, in presynaptic than in postsynaptic MSNs. Evoked corticostriatal responses are shorter and peak earlier in striatopallidal than used for $\boldsymbol{B}$ and $\boldsymbol{C}$.
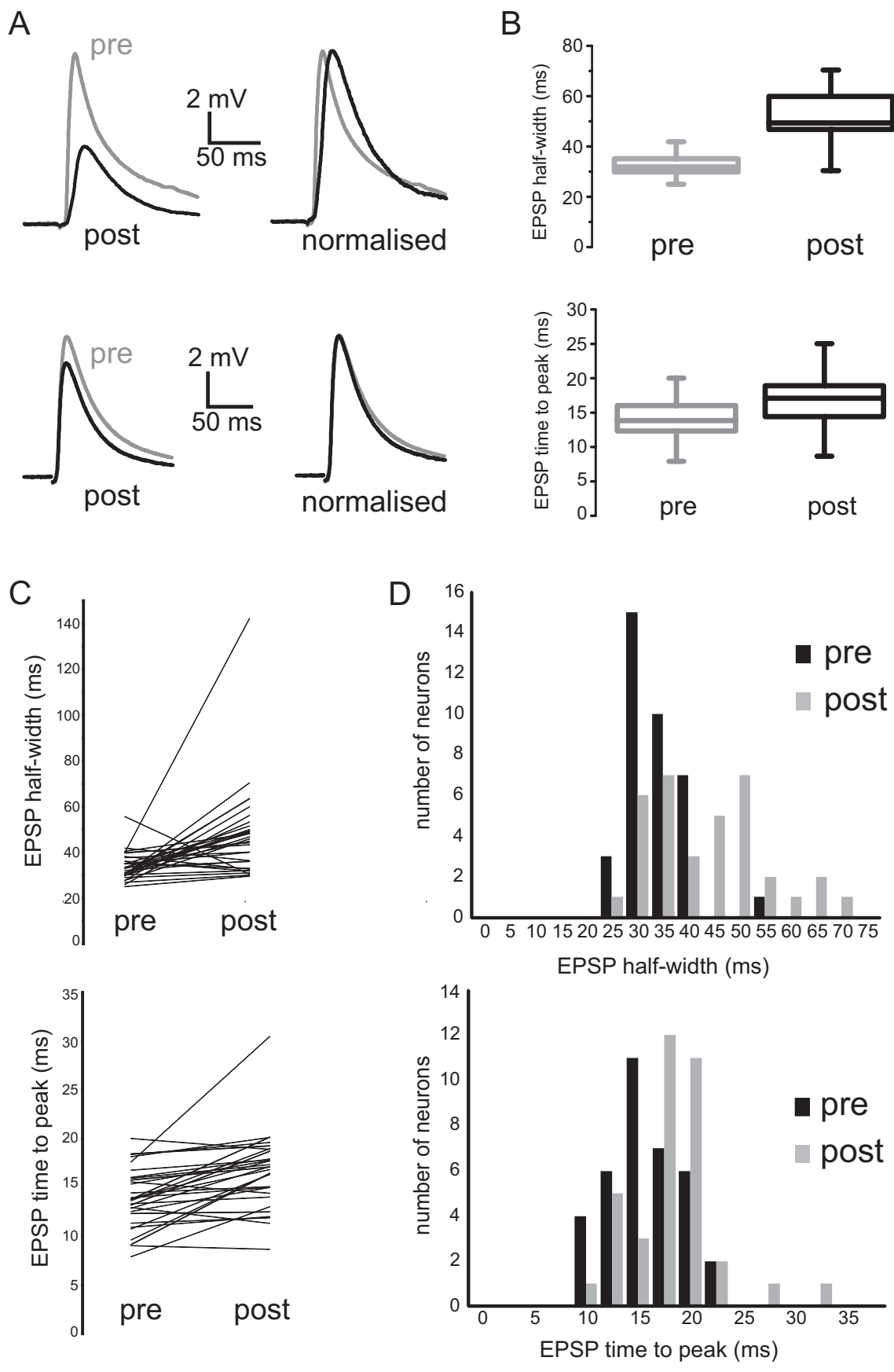

Figure 10. Corticostriatal responses differ in presynaptic and postsynaptic MSNs. A, Examples of evoked EPSPs in two different MSNs pairs. Average responses for each MSN (in the absence of spikes in the other neuron) are superimposed. In each pair, the presynaptic MSN was defined as one whose spikes that depressed the response of the other MSN. In these two experiments only interactions in one direction were present. In the first case (top), the EPSP time-to-peak and half-width were larger in the postsynaptic MSN. In the second case (bottom), similar values were observed in the two MSNs. $\boldsymbol{B}$, Box plots for evoked EPSP half-width and time-to-peak in presynaptic and postsynaptic MSNs in 31 paired recording experiments in which monodirectional interactions were present. Both half-width and time-to-peak were significantly different in presynaptic and postsynaptic MSNs ( $p=0.002$ in both cases). In box plots, the central line represents the median, the edges of the box represents the interquartile range, and the 'whisker lines' show the extent of the overall distribution. C, For each experiment, data points representing average EPSP half-width (top) and EPSP time to peak (bottom) for presynaptic and postsynaptic MSNs are connected by a line. $D$, The distribution of evoked EPSP half-width (top) and time-to-peak (bottom) in presynaptic and postsynaptic MSNs in the experiments

striatonigral MSNs (Flores-Barrera et al., 2010). Thus, it seems most likely that in our paired recoding experiments the presynaptic cells were exclusively striatopallidal MSNs, while the postsynaptic cells were a mixture of striatopallidal and striatonigral MSNs. These re- 


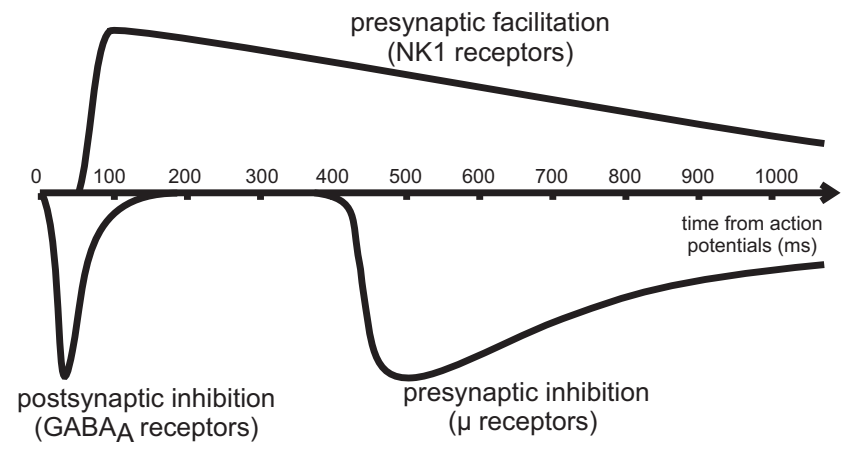

Figure 11. Time course of facilitatory and inhibitory interactions between striatal MSNs. Based on present results as well as on previously published material, we have drawn the approximate time course of the interactions mediated by $\mathrm{GABA}_{A}$, NK1 and $\mu$-opioid receptors after a brief burst of spikes in a presynaptic MSN. This presynaptic MSN is supposed to be either a striatopallidal (for MOR-mediated interactions) or a striatonigral cell (for NK1 receptormediated interactions).

sults are consistent with previous anatomical and physiological studies showing that GABAergic interactions between MSNs are not segregated in terms of the two neuronal subpopulations (Yung et al., 1996; Taverna et al., 2008).

What was the probability of connection between a certain striatopallidal cell and a nearby MSN? Assuming equal probability of connection to a postsynaptic striatopallidal or striatonigral MSN, and an equal density for the MSNs of the two populations, it is expected that approximately one quarter of all recorded pairs were formed by two striatonigral MSNs (no interactions), one quarter by two striatopallidal MSNs (interactions possible in both directions) and half by a striatopallidal and a striatonigral MSN (interactions possible in one direction). Interactions were found in $54 \%$ of pairs, and $8.9 \%$ of pairs were reciprocally connected; using simple probability calculations, one gets an estimate of $65 \%$ for the probability that a certain striatopallidal MSN connects to generic MSN located within $100 \mu \mathrm{m}$.

The strength of opioid-mediated presynaptic connections was also revealed by paired recording experiments: a brief burst of five spikes in a single MSN depressed the corticostriatal responses of a neighboring MSN by up to $30 \%$, the average inhibition being $\sim 17 \%$. These are substantial effects that are expected to affect the firing pattern of MSNs dramatically, especially as these neurons are not spontaneously active and rely entirely on glutamatergic inputs for their activation (Nisenbaum et al., 1994). We also obtained quantitative information about the time course of the opioidergic interactions; combining results from single and paired recordings we can conclude that, following a brief burst of spikes, inhibition of corticostriatal responses was absent at $250 \mathrm{~ms}$, peaked at $\sim 500 \mathrm{~ms}$ and then decayed in an approximately exponential manner, being over at 2-3 s (Fig. 11).

The present results complement our recent finding that substance P-expressing MSNs facilitate the cortical inputs to other MSN by activating presynaptic NK1 (substance P) receptors (Blomeley et al., 2009). Collectively, these results cast light on the poorly understood function of the neuropeptides expressed by MSNs. The picture emerging for the local network of MSN axon collaterals is one where fast, direct inhibitory interactions between MSNs are mediated by postsynaptic GABA contacts, while slower interactions, either facilitatory or inhibitory in nature, are mediated by substance P or enkephalin, respectively. Opioidmediated inhibition of corticostriatal inputs was found to be slightly slower and more prominent (in terms of connectivity and magnitude of the effects) than substance P-mediated facilitation. The approximate time course of these three forms of communication, as demonstrated by present and past experiments, is illustrated in Figure 11.

The original discovery that MSNs form reciprocal GABAergic synapses led to the idea that striatal networks are governed by competitive dynamics and that such dynamics may underlie vital processes such as recognition of cortical patterns and action selection (Redgrave et al., 1999; Wilson, 2000; Tunstall et al., 2002). However, different opinions exist about the ability of the relatively weak GABAergic connections between MSNs to enforce such dynamics (Wickens et al., 2007a; Wilson, 2007). The present results indicate that slower but vigorous competitive dynamics may be imposed on the striatal circuits by opioidergic inhibition of MSN cortical inputs by other MSNs. This feature coexists with the cooperative dynamics created by substance P-mediated facilitation of glutamate release (Blomeley et al., 2009). It is important that the quantitative data emerging from these experiments are incorporated into the computational models of striatal microcircuitry alongside the other known neuronal interactions, as this may reveal new emerging network properties that may cast light on striatal operation.

Further studies will be required to characterize in greater detail what determines the presence of GABAergic and peptidergic connections between MSNs as a function of their position in the striatal mosaic and the nature of their synaptic inputs and outputs. The resulting picture will then have to be integrated with the interactions mediated by the different types of striatal interneurons (Pisani et al., 2007; Tepper et al., 2010).

Exposure to opioids increases the threshold for pain; furthermore, patients with lesions of the striatum have decreased pain sensitivity (Starr et al., 2011). It has been proposed that striatal dynamics are crucial for attention (Cools, 2011); thus, it is tempting to speculate that the competitive dynamics created by opioid-mediated inhibition between MSNs play a role in pain processing, possibly by allowing painful stimuli to access attentional resources. The present results have also potential implications for drug addiction. The presynaptic MORs activated by MSNs will also be activated by exogenous opiates in heroin- or morphine-addicted subjects. While the dorsal striatum is not crucially involved in the acute rewarding effects of these drugs of abuse, its role in the formation of aberrant memories and compulsive habits in drug-addicted subjects is increasingly recognized (Everitt et al., 2008). Overstimulation of presynaptic MORs in the striatum of opioid-addicted subjects is expected to cause homeostatic changes in the density and/or efficacy of these receptors. This may in turn impair normal competitive dynamics between MSNs and disrupt striatal information processing and corticostriatal long-term plasticity (Di Filippo et al., 2009; Surmeier et al., 2009), resulting in the storage of anomalous procedural and contextual memories.

\section{References}

Alexander GE, Crutcher MD (1990) Functional architecture of basal ganglia circuits: neural substrates of parallel processing. Trends Neurosci $13: 266-271$.

Barral J, Mendoza E, Galarraga E, Bargas J (2003) The presynaptic modulation of corticostriatal afferents by mu-opioids is mediated by $\mathrm{K}+$ conductances. Eur J Pharmacol 462:91-98.

Blomeley C, Bracci E (2008) Substance P depolarizes striatal projection neurons and facilitates their glutamatergic inputs. J Physiol 586:2143-2155.

Blomeley CP, Bracci E (2009) Serotonin excites fast-spiking interneurons in the striatum. Eur J Neurosci 29:1604-1614.

Blomeley CP, Kehoe LA, Bracci E (2009) Substance P mediates excitatory 
interactions between striatal projection neurons. J Neurosci 29:4953-4963.

Bolam JP, Izzo PN (1988) The postsynaptic targets of substance P-immunoreactive terminals in the rat neostriatum with particular reference to identified spiny striatonigral neurons. Exp Brain Res 70:361-377.

Boyes J, Bolam JP (2007) Localization of GABA receptors in the basal ganglia. Prog Brain Res 160:229-243.

Cools R (2011) Dopaminergic control of the striatum for high-level cognition. Curr Opin Neurobiol 21:402-407.

Di Filippo M, Picconi B, Tantucci M, Ghiglieri V, Bagetta V, Sgobio C, Tozzi A, Parnetti L, Calabresi P (2009) Short-term and long-term plasticity at corticostriatal synapses: implications for learning and memory. Behav Brain Res 199:108-118.

Everitt BJ, Belin D, Economidou D, Pelloux Y, Dalley JW, Robbins TW (2008) Neural mechanisms underlying the vulnerability to develop compulsive drug-seeking habits and addiction [review]. Philos Trans R Soc Lond B Biol Sci 363:3125-3135.

Flores-Barrera E, Vizcarra-Chacón BJ, Tapia D, Bargas J, Galarraga E (2010) Different corticostriatal integration in spiny projection neurons from direct and indirect pathways. Front Syst Neurosci 4:15.

Gerfen CR (1992) The neostriatal mosaic: multiple levels of compartmental organization in the basal ganglia. Annu Rev Neurosci 15:285-320.

Gertler TS, Chan CS, Surmeier DJ (2008) Dichotomous anatomical properties of adult striatal medium spiny neurons. J Neurosci 28:1081410824.

Graveland GA, DiFiglia M (1985) The frequency and distribution of medium-sized neurons with indented nuclei in the primate and rodent neostriatum. Brain Res 327:307-311.

Graybiel AM, Aosaki T, Flaherty AW, Kimura M (1994) The basal ganglia and adaptive motor control. Science 265:1826-1831.

Guzmán JN, Hernández A, Galarraga E, Tapia D, Laville A, Vergara R, Aceves J, Bargas J (2003) Dopaminergic modulation of axon collaterals interconnecting spiny neurons of the rat striatum. J Neurosci 23:8931-8940.

Jiang ZG, North RA (1992) Pre- and postsynaptic inhibition by opioids in rat striatum. J Neurosci 12:356-361.

Kincaid AE, Wilson CJ (1996) Corticostriatal innervation of the patch and matrix in the rat neostriatum. J Comp Neurol 374:578-592.

Kita T, Kita H, Kitai ST (1984) Passive electrical membrane properties of rat neostriatal neurons in an in vitro slice preparation. Brain Res 300: $129-139$.

Koos T, Tepper JM, Wilson CJ (2004) Comparison of IPSCs evoked by spiny and fast-spiking neurons in the neostriatum. J Neurosci 24: $7916-7922$.

Lovinger DM (2010) Neurotransmitter roles in synaptic modulation, plasticity and learning in the dorsal striatum. Neuropharmacology 58:951961.

Miura M, Saino-Saito S, Masuda M, Kobayashi K, Aosaki T (2007) Compartment-specific modulation of GABAergic synaptic transmission by mu-opioid receptor in the mouse striatum with green fluorescent protein-expressing dopamine islands. J Neurosci 27:9721-9728.

Nisenbaum ES, Xu ZC, Wilson CJ (1994) Contribution of a slowly inactivating potassium current to the transition to firing of neostriatal spiny projection neurons. J Neurophysiol 71:1174-1189.

Pakhotin P, Bracci E (2007) Cholinergic interneurons control the excitatory input to the striatum. J Neurosci 27:391-400.

Pisani A, Bernardi G, Ding J, Surmeier DJ (2007) Re-emergence of striatal cholinergic interneurons in movement disorders. Trends Neurosci 30:545-553.

Redgrave P, Gurney K (2006) The short-latency dopamine signal: a role in discovering novel actions? Nat Rev Neurosci 7:967-975.
Redgrave P, Prescott TJ, Gurney K (1999) The basal ganglia: a vertebrate solution to the selection problem? Neuroscience 89:1009-1023.

Shindou T, Arbuthnott GW, Wickens JR (2008) Actions of adenosine A2A receptors on synaptic connections of spiny projection neurons in the neostriatal inhibitory network. J Neurophysiol 99:1884-1889.

Smeal RM, Gaspar RC, Keefe KA, Wilcox KS (2007) A rat brain slice preparation for characterizing both thalamostriatal and corticostriatal afferents. J Neurosci Methods 159:224-235.

Starr CJ, Sawaki L, Wittenberg GF, Burdette JH, Oshiro Y, Quevedo AS, McHaffie JG, Coghill RC (2011) The contribution of the putamen to sensory aspects of pain: insights from structural connectivity and brain lesions. Brain 134:1987-2004.

Surmeier DJ, Plotkin J, Shen W (2009) Dopamine and synaptic plasticity in dorsal striatal circuits controlling action selection. Curr Opin Neurobiol 19:621-628.

Taverna S, Ilijic E, Surmeier DJ (2008) Recurrent collateral connections of striatal medium spiny neurons are disrupted in models of Parkinson's disease. J Neurosci 28:5504-5512.

Tepper JM, Wilson CJ, Koós T (2008) Feedforward and feedback inhibition in neostriatal GABAergic spiny neurons. Brain Res Rev 58:272-281.

Tepper JM, Tecuapetla F, Koós T, Ibáñez-Sandoval O (2010) Heterogeneity and diversity of striatal GABAergic interneurons. Front Neuroanat 4:150.

Tunstall MJ, Oorschot DE, Kean A, Wickens JR (2002) Inhibitory interactions between spiny projection neurons in the rat striatum. J Neurophysiol 88:1263-1269.

Wang H, Pickel VM (1998) Dendritic spines containing mu-opioid receptors in rat striatal patches receive asymmetric synapses from prefrontal corticostriatal afferents. J Comp Neurol 396:223-237.

Wang H, Pickel VM (2001) Preferential cytoplasmic localization of deltaopioid receptors in rat striatal patches: comparison with plasmalemmal mu-opioid receptors. J Neurosci 21:3242-3250.

Wang Z, Kai L, Day M, Ronesi J, Yin HH, Ding J, Tkatch T, Lovinger DM, Surmeier DJ (2006) Dopaminergic control of corticostriatal long-term synaptic depression in medium spiny neurons is mediated by cholinergic interneurons. Neuron 50:443-452.

Wee S, Koob GF (2010) The role of the dynorphin-kappa opioid system in the reinforcing effects of drugs of abuse. Psychopharmacology (Berl) 210:121-135.

Wickens JR, Arbuthnott GW, Shindou T (2007a) Simulation of GABA function in the basal ganglia: computational models of GABAergic mechanisms in basal ganglia function. Prog Brain Res 160:313-329.

Wickens JR, Budd CS, Hyland BI, Arbuthnott GW (2007b) Striatal contributions to reward and decision making: making sense of regional variations in a reiterated processing matrix. Ann N Y Acad Sci 1104:192-212.

Wilson CJ (2000) Striatal circuitry: Categorically selective, or selectively categorical? In: Brain dynamics and the striatal complex (Miller RH, Wickens J, eds), pp 289-305. New York: Taylor \& Francis.

Wilson CJ (2007) GABAergic inhibition in the neostriatum. Prog Brain Res 160:91-110.

Wilson CJ, Groves PM (1980) Fine structure and synaptic connections of the common spiny neuron of the rat neostriatum: a study employing intracellular inject of horseradish peroxidase. J Comp Neurol 194:599615.

Wilson CJ, Chang HT, Kitai ST (1983) Origins of post synaptic potentials evoked in spiny neostriatal projection neurons by thalamic stimulation in the rat. Exp Brain Res 51:217-226.

Yung KK, Smith AD, Levey AI, Bolam JP (1996) Synaptic connections between spiny neurons of the direct and indirect pathways in the neostriatum of the rat: evidence from dopamine receptor and neuropeptide immunostaining. Eur J Neurosci 8:861-869. 